\title{
Insertion Angle of Pedicle Screws in the Subaxial Cervical Spine: The Analysis of Computed Tomography-Navigated Insertion of Pedicle Screws
}

\author{
Stavros Oikonomidis ${ }^{*}$ Frank Beyer ${ }^{*}$, Carolin Meyer, Christoph Tobias Baltin, Peer Eysel, Jan Bredow \\ Department of Orthopedics and Trauma Surgery, Faculty of Medicine and University Hospital Cologne, University of Cologne, Cologne, Germany
}

Study Design: Four orthopedic spine surgeons measured the radiological parameters of pedicle screws in the cervical spine using a postoperative computed tomography (CT) scan.

Purpose: This study analyzed the insertion angle of CT-navigated insertion of pedicle screws in the subaxial cervical spine and classified them according to their position.

Overview of Literature: Overall, a pedicle transverse angle of $33.6^{\circ}-50.2^{\circ}$ with a mean angle of $45^{\circ}$ relative to the midline has been reported in the literature.

Methods: The insertion angles of 87 pedicle screws inserted using CT-based navigation in the subaxial cervical spine were measured in the postoperative CT. The screw positioning was determined according to the modified Gertzbein and Robbins classification.

Results: Total 89.3\% ( $n=78$ ) of the pedicle screws inserted using CT-based navigation showed good placement. The mean insertion angle of the pedicle screws that showed good positioning was $29.9^{\circ} \pm 9.9^{\circ}$. The pedicle screws showing bad positioning had a mean insertion angle of $26.8^{\circ} \pm 10.5^{\circ}(p=0.157)$. The interobserver reliability showed a reliable measurement intraclass correlation coefficient: 0.994 (95\% confidence interval, 0.992-0.996).

Conclusions: The present results show that the insertion angle of the pedicle screws in the subaxial cervical spine was smaller than the actual pedicle transverse angle, as per the literature. One reason for this discrepancy could be that the navigation systems allow the insertion of cervical pedicle screws with a lower convergence.

Keywords: Cervical spine; Pedicle transverse angle; Navigation; Pedicle screws; Insertion angle

\section{Introduction}

Posterior instrumentation of the subaxial cervical spine is a common procedure for the treatment of many degenerative conditions, fractures, and tumor diseases of the cervical spine $[1,2]$. Transpedicular screw fixation provides sufficient stability in the cervical spine and in multilevel fixation under torsion and extension loading [3]. This stability depends on the biomechanical investigations superior to conventional cervical fixation systems such as anterior vertebral screws and posterior triple wiring [3]. However, major complications during the insertion of cervical pedicle screws have been reported in the literature, such as vertebral artery and nerve root injury

Received Feb 17, 2019; Revised Apr 20, 2019; Accepted Apr 24, 2019

Corresponding author: Stavros Oikonomidis

Department of Orthopedics and Trauma Surgery, Faculty of Medicine and University Hospital Cologne, University of Cologne, Kerpener Str. 62, 50937 Cologne, Gemany

Tel: +49-1724254544, Fax: +49-221-478 6060, E-mail: Stavros.oikonomidis@uk-koeln.de

*These two authors contributed equally to this work. 

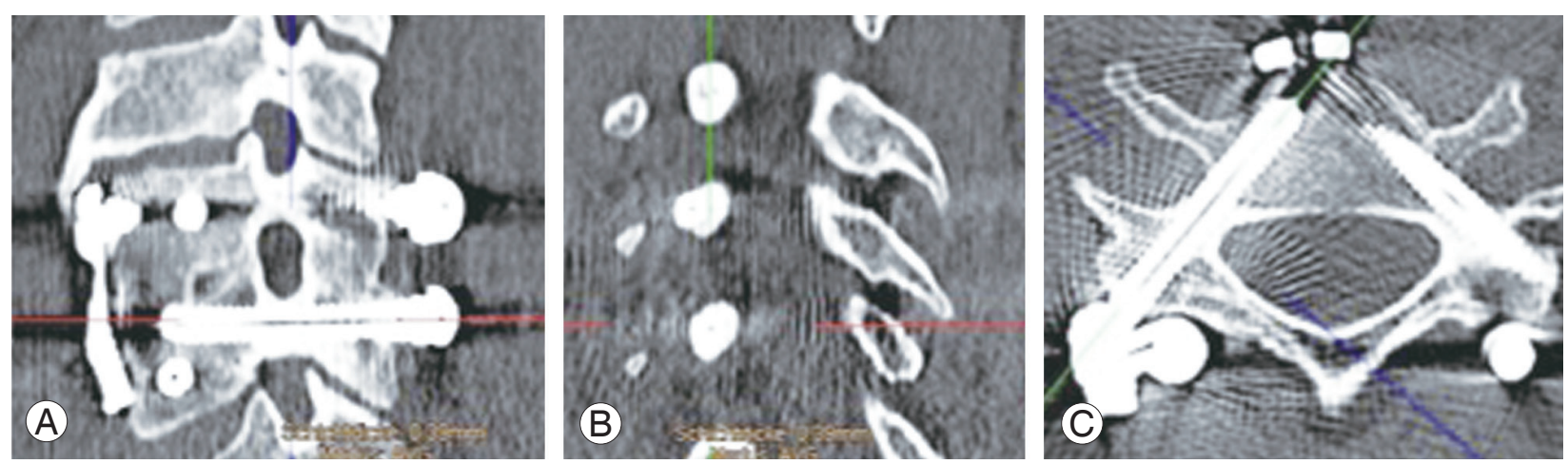

Fig. 1. (A-C) Computed tomography-navigated insertion of pedicle screw classified as grade 1 according to the modified Gertzbein and Robbins classification.

[4]. With the introduction of navigation systems, pedicle screw insertion in the subaxial cervical spine has become safer and more accurate than two-dimensional fluoroscopy pedicle screw placement [5]. Nonetheless, even with the use of navigation systems, the overall accuracy of cervical pedicle screw insertion is reported to be $88.5 \%$ [6]. Therefore, precise knowledge of the anatomical landmarks and directions for screw insertion is essential. Higher accuracy has been reported in studies using $\mathrm{O}$-arm based three-dimensional navigation for the insertion of cervical pedicle screws [7].

Abumi et al. [2] first described the landmarks and direction of the screw needed for successful insertion. Based on the data of 13 patients, the authors suggested that the insertion angle should be $30^{\circ}-40^{\circ}$ medial to the midline in the transverse plane [2]. Further investigations have identified the transverse angle for safe insertion of a pedicle screw in the subaxial cervical spine. Overall, a pedicle transverse angle of $33.6^{\circ}-50.2^{\circ}$ with a mean angle of $45^{\circ}$ relative to the midline has been reported in the literature [8-11].

This study aimed to analyze the insertion angle of computed tomography (CT)-navigated insertion of pedicle screws in the subaxial cervical spine and classify them as per the accuracy of their positioning. Furthermore, we aimed to compare the in-vivo-pedicle insertion angle of the pedicle screws with the recommendations given in previous trials.

\section{Materials and Methods}

\section{Study design}

We retrospectively collected the data of 28 patients who
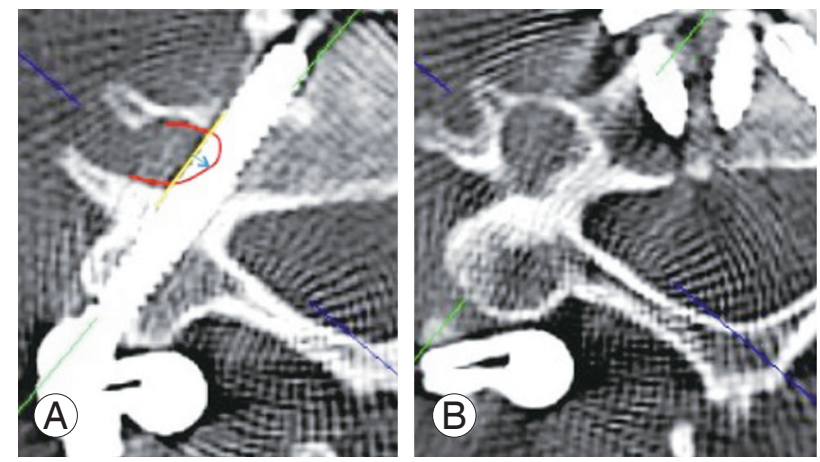

Fig. 2. (A, B) Computed tomography-navigated insertion of pedicle screw classified as grade 2 according to the modified Gertzbein and Robbins classification.

underwent dorsal CT-navigated cervical (C3-C7) pedicle instrumentation. The patient inclusion criteria were as follows: CT-navigated insertion of subaxial cervical pedicle screws and a postoperative multiplanar CT scan of the cervical spine. Total 87 pedicle screws could be identified. Screw positioning and the transverse angle of the pedicle screws were evaluated individually by four orthopedic surgeons who specialize in spine surgery. The evaluation of screw positioning was performed as per the modified Gertzbein and Robbins classification consisting of five grades [12]. Grade 1 describes ideal screw position with pedicle wall perforation of $1 \mathrm{~mm}$ (Fig. 1), grade 2 describes positioning with pedicle wall perforation of 2 mm (Fig. 2), grade 3 of $3 \mathrm{~mm}$ (Fig. 3), and grade 4 of 4 $\mathrm{mm}$. Grade 5 represents a cortical breach of $4 \mathrm{~mm}$ and/ or obstruction of the transverse foramen by more than half a screw diameter (Fig. 4). The screw positioning was defined as "good" when the grade was 1 or 2 and "bad" when the grade was 3,4 , or 5 .

The insertion angle of the pedicle screw was determined as the angle between the long axis of the pedicle screws 

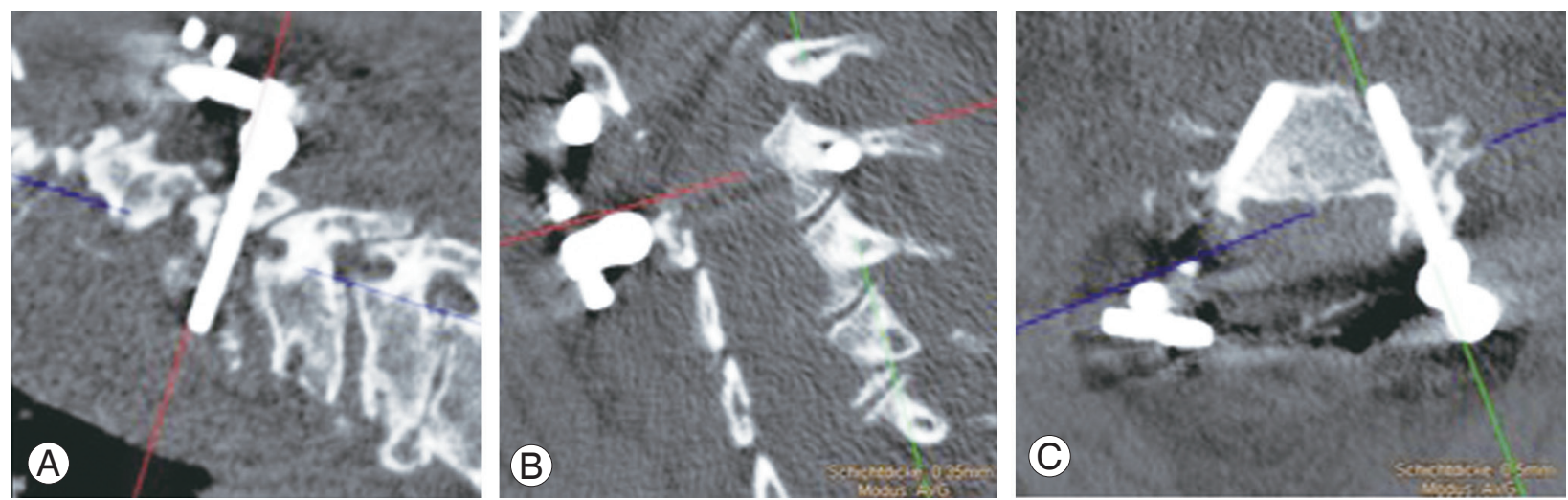

Fig. 3. (A-C) Computed tomography-navigated insertion of pedicle screw classified as grade 3 according to the modified Gertzbein and Robbins classification.
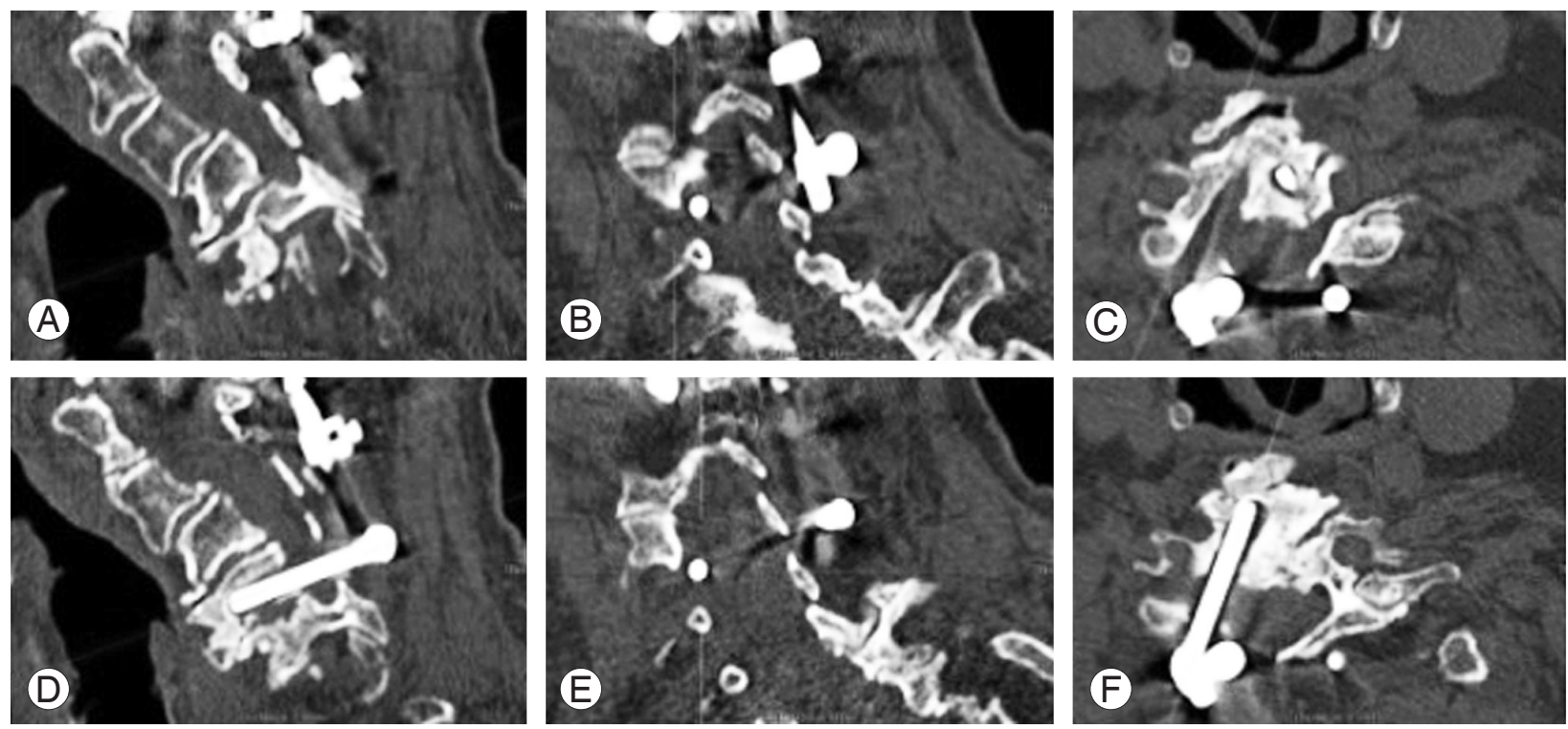

Fig. 4. (A-F) Computed tomography-navigated insertion of pedicle screw classified as grade 5 according to the modified Gertzbein and Robbins classification.

and the midline of the vertebral body in the transversal plane [10]. The mean transverse angle of the pedicle screws was assessed.

Four orthopedic spine surgeons measured all the parameters using basic functions in IMPAX EE (Agfa HealthCare GmbH, Bonn, Germany), running in a $\mathrm{Mi}-$ crosoft Windows environment. Demographic data of the patients were collected from the electronic patient records.

\section{Statistical analyses}

Statistical analyses were performed using IBM SPSS ver. 25.0 software (IBM Corp., Armonk, NY, USA). Descrip- tive statistics were used to calculate the mean values of demographic data, transverse angle, and screw position. Intraclass correlation coefficient (ICC) values were assessed in a two-way mixed model with absolute agreement at $95 \%$ confidence intervals for interobserver reliability. Values $<0.40$ were considered poor, those between 0.40 and 0.59 were considered fair, those from 0.60 to 0.74 were considered good, and those between 0.75 and 1.00 were considered excellent [13]. The level of significance was set at 0.05 .

Institutional Review Board approval was not necessary because of the retrospective design of the study. Written informed consents of the patients was obtained. 


\section{Results}

\section{Demographics and clinical data}

The CT images of 28 patients were analyzed. Eight patients were women, and 20 were men. The average age of the patients was $57.8 \pm 18.3$ years (range, $19-85$ years). The reason for performing instrumentation was trauma in 16 cases (57.1\%), degeneration in three cases (10.7\%), tumor in six cases (21.4\%), rheumatoid arthritis in two cases (7.1\%) and vertebral osteomyelitis in one case (3.6\%). The distribution of the cervical spine levels is described in Table 1.

The mean operation time was $240.4 \pm 88.7$ minutes (range, 107-518 minutes). A perioperative complication, such as incidental durotomy, occurred in one case (3.6\%), resulting in a liquor fistula. A postoperative complication was documented in five cases. In four cases (14.3\%), a wound-healing disorder was reported, and a postoperative vertebral osteomyelitis was reported in one case (3.6\%).

\section{Positioning and insertion angle of the pedicle screws}

Total 87 pedicle screws were assessed. The detailed classification of the position of the screws is described in Table 2.

Table 1. Distribution of the instrumented cervical vertebral bodies

\begin{tabular}{lc} 
Cervical vertebrae & No. $(\%)$ \\
C3 & $6(6.9)$ \\
C4 & $8(9.2)$ \\
C5 & $22(25.3)$ \\
C6 & $23(26.4)$ \\
C7 & $28(32.2)$ \\
Total & $87(100.0)$ \\
\hline
\end{tabular}

Table 2. Classification of the position of the pedicle screws

\begin{tabular}{lc} 
Modified Gertzbein and Robbins classification & No. $(\%)$ \\
\hline 1 & $32(36.8)$ \\
2 & $37(42.5)$ \\
3 & $9(10.3)$ \\
4 & $3(3.4)$ \\
\hline 5 & $6(6.9)$ \\
Total & $87(100.0)$ \\
\hline
\end{tabular}

We found that $89.7 \%(n=78)$ of the pedicle screws showed good positioning (grade 1 or 2). Examples of grade 1 and 2 placement are shown in Fig. 1 and Fig. 2, respectively. Further, $10.3 \%(n=9)$ of the pedicle screws showed bad positioning (grade 3, 4, or 5). Fig. 3 shows grade 3 screw placements, and Fig. 4 shows an example of grade 5 placement. The evaluation of the classification of the pedicle screws showed excellent interobserver reliability, with ICC 0.991 (95\% confidence interval, 0.988-0.994).

The mean insertion angle of the pedicle screws that showed good positioning was $29.9^{\circ} \pm 9.9^{\circ}$. The pedicle screws showing bad positioning had a mean insertion angle of $26.8^{\circ} \pm 10.5^{\circ}$. There was no statistically significant difference in the insertion angle of well and badly positioned screws $(p=0.157)$. The measurement of the insertion angles showed excellent interobserver reliability, with ICC 0.994 (95\% confidence interval, 0.992-0.996).

\section{Discussion}

The present results reveal that the insertion of CT-navigated pedicle screws in the subaxial cervical spine is associated with safe placement. In total, $89.7 \%$ of the pedicle screws showed good positioning. These results correlate with the accuracy of CT-navigated insertion of subaxial pedicle screws reported in the literature [6,14]. Moreover, our results show a mean insertion angle for the pedicle screws of $29.9^{\circ} \pm 9.9^{\circ}$. However, these values differ from the mean values of the pedicle transverse angles reported in the literature. In an anatomical study of 22 human cadaver subaxial cervical spines, Mohi Eldin [15] reports about medial angulation of the pedicles ranging from $37^{\circ}-47^{\circ}$ at $\mathrm{C} 3$, from $33^{\circ}-45^{\circ}$ at $\mathrm{C} 4$, from $40^{\circ}-52^{\circ}$ at $\mathrm{C} 5$, from $37^{\circ}-42^{\circ}$ at $\mathrm{C} 6$, and from $41^{\circ}-47^{\circ}$ at $\mathrm{C} 7$. In a further investigation of CT scans of 122 cervical spines, Onibokun et al. [8] report a mean pedicle transverse angle of approximately $44^{\circ}$ from C3 to $\mathrm{C} 6$ and $37.8^{\circ}$ at C7. Similar values of pedicle transverse angles are reported by Nishinome et al. [9] based on an investigation of 50 CT scans of cervical spines (C3 to C6). They report a pedicle transverse angle of $37.1^{\circ}-45.4^{\circ}$. Chazono et al. [16] analyzed the CT scans of the cervical spine (C3-C7) of 60 patients and measured a mean pedicle transverse angle of $33.6^{\circ}-50.2^{\circ}$. Another analysis of $100 \mathrm{CT}$ scans of the cervical spines by Westermann et al. [17] reports pedicle transverse angels of $34.6^{\circ}-48.02^{\circ}$. In sum, the average cervical pedicle transverse angle reported in the studies is considerably higher 
than the insertion angle of pedicle screws reported in our study. Nevertheless, our results show reasonable accuracy of the pedicle screws. A different pedicle screw entrance point could lead to a deviation of the transverse angle of the drill and the true pedicle transverse angle. However, the trajectory may be within the cortical bone of the cervical pedicle [18]

One reason for this deviation could be that the entry point of the pedicle screws inserted with $\mathrm{CT}$ navigation is being shifted toward the medial side. A medial entry point leads to lower convergence of the insertion of the pedicle screw and lower insertion angle of the pedicle screw. To insert a cervical pedicle screw with the optimal transverse pedicle angle reported in the literature, a high convergence is necessary. High convergence for the insertion angle requires a wide incision and exposure of the soft tissue. Smaller incision and exposure of the soft tissue can possibly prevent postoperative complications, such as wound infections and healing disorders. Moreover, a smaller surgical approach leads to limited approachrelated injuries to the paraspinous muscles and posterior ligamentous complex. However, proper preparation and exposure of the anatomical landmarks in the cervical spine is essential for safe pedicle screw insertion even when using navigation. Surgeons should be aware of the anatomical landmarks and should not rely only on navigation for insertion. The exposure and identification of the anatomical entry points should be an essential step of the operation.

Despite lower convergence for the insertion angle of the pedicle screws, CT navigation allows safe insertion, as reported in our study. Uehara et al. [10] analyzed the pre- and postoperative CT of cervical spines instrumented with pedicle screws. They report significantly smaller pedicle screw insertion angles than pedicle transverse angles for the subaxial cervical spine. They conclude that the insertion angle for safe insertion of pedicle screws should be between $24.5^{\circ}$ and $36.5^{\circ}$. Angles $<24.5^{\circ}$ can cause lateral perforation and those $>36.5^{\circ}$ can cause medial perforation. The optimal screw insertion angle is reported as $30^{\circ}$ [10]. These findings agree with our results.

\section{Conclusions}

The present results reveal that the insertion angle of pedicle screws in the subaxial cervical spine is smaller than the actual pedicle transverse angle when using CT navigation and this procedure is safe and accurate. One reason could be that the smaller insertion angle of the pedicle screws is associated with lower convergence leading to smaller incision and soft tissue exposure.

\section{Conflict of Interest}

No potential conflict of interest relevant to this article was reported.

\section{Author Contributions}

Stavros Oikonomidis contributed to the writing of the paper, to searching for references, data collection and data analysis. Jan Bredow, Frank Beyer, Carolin Meyer, and Christoph Tobias Baltin measured the insertion angles of the pedicle screws and contributed to the data analysis. Peer Eysel contributed to the study design. All authors reviewed and approved the final submitted version of the paper.

\section{References}

1. Abumi K, Kaneda K. Pedicle screw fixation for nontraumatic lesions of the cervical spine. Spine (Phila Pa 1976) 1997;22:1853-63.

2. Abumi K, Itoh H, Taneichi H, Kaneda K. Transpedicular screw fixation for traumatic lesions of the middle and lower cervical spine: description of the techniques and preliminary report. J Spinal Disord 1994;7:19-28.

3. Kotani Y, Cunningham BW, Abumi K, McAfee PC. Biomechanical analysis of cervical stabilization systems: an assessment of transpedicular screw fixation in the cervical spine. Spine (Phila Pa 1976) 1994;19:2529-39.

4. Abumi K, Shono Y, Ito M, Taneichi H, Kotani Y, Kaneda K. Complications of pedicle screw fixation in reconstructive surgery of the cervical spine. Spine (Phila Pa 1976) 2000;25:962-9.

5. Ishikawa Y, Kanemura T, Yoshida G, Ito Z, Muramoto A, Ohno S. Clinical accuracy of three-dimensional fluoroscopy-based computer-assisted cervical pedicle screw placement: a retrospective comparative study of conventional versus computer-assisted cervical pedicle screw placement. J Neurosurg Spine 2010;13:606-11. 
6. Rienmuller A, Buchmann N, Kirschke JS, et al. Accuracy of CT-navigated pedicle screw positioning in the cervical and upper thoracic region with and without prior anterior surgery and ventral plating. Bone Joint J 2017;99-B:1373-80.

7. Chachan S, Bin Abd Razak HR, Loo WL, Allen JC, Shree Kumar D. Cervical pedicle screw instrumentation is more reliable with $\mathrm{O}$-arm-based $3 \mathrm{D}$ navigation: analysis of cervical pedicle screw placement accuracy with O-arm-based 3D navigation. Eur Spine J 2018;27:2729-36.

8. Onibokun A, Khoo LT, Bistazzoni S, Chen NF, Sassi M. Anatomical considerations for cervical pedicle screw insertion: the use of multiplanar computerized tomography measurements in 122 consecutive clinical cases. Spine J 2009;9:729-34.

9. Nishinome M, Iizuka H, Iizuka Y, Takagishi K. An analysis of the anatomic features of the cervical spine using computed tomography to select safer screw insertion techniques. Eur Spine J 2013;22:2526-31.

10. Uehara M, Takahashi J, Ikegami S, et al. Optimal cervical screw insertion angle determined by means of computed tomography scans pre- and postoperatively. Spine J 2017;17:190-5.

11. Jones EL, Heller JG, Silcox DH, Hutton WC. Cervical pedicle screws versus lateral mass screws: anatomic feasibility and biomechanical comparison. Spine
(Phila Pa 1976) 1997;22:977-82.

12. Gertzbein SD, Robbins SE. Accuracy of pedicular screw placement in vivo. Spine (Phila Pa 1976) 1990;15:11-4.

13. Lachin JM. The role of measurement reliability in clinical trials. Clin Trials 2004;1:553-66.

14. Bredow J, Oppermann J, Kraus B, et al. The accuracy of 3D fluoroscopy-navigated screw insertion in the upper and subaxial cervical spine. Eur Spine J 2015;24:2967-76.

15. Mohi Eldin MM. Cervical pedicle screw fixation: anatomic feasibility of pedicle morphology and radiologic evaluation of the anatomical measurements. Asian Spine J 2014;8:273-80.

16. Chazono M, Soshi S, Inoue T, Kida Y, Ushiku C. Anatomical considerations for cervical pedicle screw insertion: the use of multiplanar computerized tomography reconstruction measurements. J Neurosurg Spine 2006;4:472-7.

17. Westermann L, Spemes C, Eysel P, et al. Computer tomography-based morphometric analysis of the cervical spine pedicles C3-C7. Acta Neurochir (Wien) 2018;160:863-71.

18. Wang Y, Xie J, Yang Z, et al. Computed tomography assessment of lateral pedicle wall perforation by freehand subaxial cervical pedicle screw placement. Arch Orthop Trauma Surg 2013;133:901-9. 